\title{
The Aftermath of COVID-19 Lockdown on Daily Life Activities in Orthopaedic Patients
}

This article was published in the following Dove Press journal:

Journal of Pain Research

\author{
Pietro Persiani ${ }^{1}$ \\ Daniele De Meo (D) ${ }^{\prime}$ \\ Elettra Giannini' \\ Valeria Calogero' \\ Tommaso Speziale Varsamis ${ }^{\prime}$ \\ Armando Ugo Cavallo ${ }^{2}$ \\ Lorena Martini' \\ Gianluca Cera' \\ Flaminia Coluzzi (iD) ${ }^{3}$ \\ Ciro Villani' \\ 'Department of Orthopaedic and \\ Traumatology, Policlinico Umberto \\ I Hospital, Sapienza University of Rome, \\ Rome, 00185, Italy; ${ }^{2}$ Department of \\ Biomedicine and Prevention, Tor Vergata \\ University, Rome, 00133, Italy; \\ ${ }^{3}$ Department of Medical and Surgical \\ Sciences and Biotechnologies, Sapienza \\ University of Rome, Rome, 00185, Italy
}

Correspondence: Daniele De Meo Department of Orthopaedic and

Traumatology, Policlinico Umberto I Hospital, Sapienza University of Rome,

Piazzale A. Moro 3, Rome, 00185, Italy

Tel +393338745373

Email daniele.demeo@uniromal.it
Purpose: Following the 2019 coronavirus (COVID-19) pandemic, many reports have investigated the psychosocial effects of the lockdown. The aim of our study was to investigate the effects of the lockdown on the daily life habits of orthopaedic patients.

Patients and Methods: Around 30 days after the start of the Italian lockdown, from April 17th to the end of the containment measures on May 18th, the Sapienza University of Rome carried out a 19-item survey on the effects the lockdown caused in patients suffering from an orthopaedic pathology (with regard to changes in lifestyle, pain, treatment) by stratifying the sample by age, sex, profession, pain location, pathogenesis and prior surgeries. Results: A total of 292 subjects with orthopaedic pathologies took part in the survey. Taking into consideration the totality of the sample, physical activity was significantly reduced ( $p$ $<0.001$ ). The increase of a sedentary lifestyle was statistically significant ( $p<0.001)$, as well as an increase in pain $(\mathrm{p}<0.001)$ and the need to adjust the pharmacological treatment $(\mathrm{p}$ $<0.001$ ). The increase in pain was particularly significant in the 50-70 age group, among workers, and in polyarthralgic patients. About 55.48\% stated that the lockdown period worsened their underlying pathological condition and $53.77 \%$ expressed the need to undergo a new clinical evaluation at the end of the lockdown.

Conclusion: The impact of the COVID-19 pandemic lockdown and resulting sudden changes in lifestyle led to a change in patient perception of orthopedic pathology including a worsening of musculoskeletal pain requiring an adjustment or change in pharmacological and physical therapy.

Keywords: lockdown, musculoskeletal pain, orthopaedic pathology, SARS-CoV-2, pandemic

\section{Introduction}

\section{Background}

December 2019, in the city of Wuhan - China, saw the beginning of a highly contagious pneumonia epidemic of unknown origin, which led local health authorities to implement containment measures. The etiological agent isolated in early January $2020^{22}$ was a new coronavirus called "severe acute respiratory syndrome" (SARS) Coronavirus (CoV) -2-related (SARS-CoV-2). ${ }^{9}$ The WHO General Director declared this infection pandemic on March 11th, 2020 (at www.who.int/ emergencies).

Italy was the first European country involved and one of the first to establish a lockdown and social distancing measures. The entire country was declared officially on lockdown on March 10th. Italy was quarantined and the government instituted stronger containment measures. ${ }^{10}$ The tight containment measures 
imposed from March 11th, by means of two consecutive government decrees, severely limited the country's productive activities alongside its citizen's personal ones. All educational activities and all commercial businesses were closed; where possible, many offices adopted smartworking measures to allow employees to work from their home computers. Physical activity was allowed only if performed solo, close to home, while keeping an adequate distance from other people.

From May 4th, once a decrease in the spread of the epidemic was obtained, some of the restrictions were lifted with a partial and gradual reopening of commercial establishments, and the population resumed moving and relating with one another.

\section{Rationale}

The lockdown resulted in a radical change of the daily habits of the entire population. Various studies have been conducted to evaluate the effects of the lockdown on existing habits and pathologies. The majority of these studies concerned the psychological aspects and psychosocial dynamics deriving from the situation itself. ${ }^{1-3,7,21}$

These studies show how the drastic increase of stress, anxiety, insomnia, and uncertainty for economic prospects significantly affects mental health and that therefore mental health management should be a focus point.

Amerio et al conducted a web-based survey that exposes a worsening of quality of life in both mental and physical component. $^{2}$

Other studies dealt with unemployment and health consequences, especially in countries with private healthcare systems. ${ }^{4,5}$ Another aspect of primary interest was the reductions in atmospheric pollution as a result of the drastic decrease in cars circulating both in China and Italy. ${ }^{6,13}$ There have been very few studies concerning the change of habits regarding physical activity.

Goethals et al conducted a qualitative survey that shows how older people expressed the need to perform physical activity at home, but that at the same time they are concerned about the risk of injury due to a lack of suitable equipment or poor knowledge of the physical exercises to perform. ${ }^{8}$

Narici et al in their study highlighted that the deleterious effects of inactivity can be diminished by routine exercise practice in home-settings, especially if combined with a reduction in calorie intake. ${ }^{15}$

The aim of our study was to investigate the effects of the lockdown on people suffering from orthopaedic pathologies priorly to the spread of the virus - in terms of lifestyle changes, pain, variations of the prescribed therapy - through the administration of a questionnaire. Our hypothesis was that the COVID-19 lockdown had negative effects on all of the aspects mentioned above.

\section{Patients and Methods}

Following the entry into force of the lockdown on 9/03/ 2020, the Department of Orthopaedics and Traumatology of the La Sapienza University of Rome conducted this observational, descriptive, cross-sectional study. To this end, a survey was launched, in compliance with the laws in force on privacy (EU Regulation GDPR 2016/679) and in accordance with the principles of the Declaration of Helsinki, the participation of which was on a voluntary basis. The questionnaire, posted on the university institutional website (https://www.uniroma1.it/ it/notizia/un-questionario-sui-problemi-ortopedici-nellafase-del-lockdown) around 30 days after the entry into force of the lockdown measures, was aimed at the general population, regardless of age, suffering from an orthopaedic pathology, both treated or undergoing treatment, of a post-traumatic or degenerative nature. The data collection terminated at the end of the lockdown period.

The questionnaire was developed with Google Forms software (Google LLC, 1600 Amphitheater Parkway Mountain View, CA 94043 USA).

In order to create a survey with targeted questions and a clear purpose, we first identified the topics of investigation, then reviewing the scientific literature and following discussion in the research group, we built concept maps. The terminology was suitable for the socio-economic characteristics of the patients involved. The anonymity was respected.

This questionnaire consisted of 19 items of which: 18 multiple choice closed-ended questions and one openended question. Of the 15 multiple-choice questions with the option of a single possible answer, 3 questions provided the possibility of giving multiple answers. Six questions were of an epidemiological nature for the purpose of demographic assessment: age group, sex, profession, location of the orthopaedic problem, nature of the current or prior pathology - ie, traumatic or degenerative and whether the subject underwent surgery or not. Ten multiple-choice questions referred to a comparison between before and after the entry into force of the lockdown. In particular: 
- 2 questions investigated the frequency with which physical activity was performed before and during the lockdown;

- 2 questions referred to the hours spent sitting/lying on the sofa/chair before and during the lockdown (defined as "couch time" in the text);

- 2 questions referred to the physiotherapy performed before and during the lockdown;

- 2 questions investigated the intake of pharmaceutical drugs before the lockdown and the need to change/ adjust their intake during the lockdown (defined as "therapy adjustment" in the text);

- lastly, they were asked to express the pain perceived before and during the lockdown, expressed through the Numeric Rating Scale (NRS).

- 2 questions referred to a self-assessment regarding any possible aggravation of one's orthopaedic pathology and the perceived need to undergo new orthopaedic check-ups.

\section{Statistic Analysis}

The statistical analysis was carried out with the software RV 3.4.4 (R Core Team (2018). R: A language and environment for statistical computing. $\mathrm{R}$ Foundation for Statistical Computing, Wien, Austria. URL https://www. r-project.org/). The continuous variables (pre- and postlockdown Pain) were analysed with the $t$-test, while the Fisher exact-test or Chi-Square test was used, where applicable, for the categorical variables (physical activity, couch time, therapy adjustment, physiotherapy). A 95\% confidence interval and a statistically significant $\mathrm{p}$-value $<0.05$ were considered for all these results.

\section{Results}

Of the 515 people who decided to participate in the questionnaire, 292 were affected by orthopaedic pathologies, which were priory treated or are under treatment, and were therefore eligible for the continuation of the survey, thus constituting our sample.

\section{Demographics of the Sample}

Table 1 shows the distribution of the sample by sex, age group, profession, anatomical districts affected by the pathology, etiopathogenesis of the orthopaedic pathology and any undergone surgery. About $57.19 \%$ of the sample was aged between 50 and 70 years, an equal distribution of men and women, with predominantly sedentary jobs
Table I Demographic Data of the Population

\begin{tabular}{|c|c|c|}
\hline Demographic & $\mathbf{N}$ & $\%$ \\
\hline \multicolumn{3}{|l|}{ Age } \\
\hline$<35$ y-o & 30 & 10.27 \\
\hline $35-50 y-0$ & 67 & 22.95 \\
\hline $50-70$ y-o & 167 & 57.19 \\
\hline$>70 y-0$ & 28 & 9.59 \\
\hline \multicolumn{3}{|l|}{ Sex } \\
\hline Male & 143 & 48.97 \\
\hline Female & 149 & 51.03 \\
\hline \multicolumn{3}{|l|}{ Occupation } \\
\hline Student & 12 & 4.11 \\
\hline Manual worker & 60 & 20.55 \\
\hline Sedentary worker & 153 & 52.40 \\
\hline Retiree & 67 & 22.95 \\
\hline \multicolumn{3}{|l|}{ Pain localization } \\
\hline Hip & 50 & 17.12 \\
\hline Knee & 40 & 13.70 \\
\hline Foot\&Ankle & 10 & 3.42 \\
\hline Shoulder & 6 & 2.05 \\
\hline Wrist\&Hand & 3 & 1.03 \\
\hline Spine & 80 & 27.40 \\
\hline Polyarthralgia & 103 & 35.27 \\
\hline \multicolumn{3}{|l|}{ Pain pathogenesis } \\
\hline Degenerative & 197 & 67.47 \\
\hline Post-traumatic & 95 & 32.53 \\
\hline \multicolumn{3}{|l|}{ Previous surgery } \\
\hline Yes & 133 & 45.55 \\
\hline No & 159 & 54.45 \\
\hline \multicolumn{3}{|l|}{ Therapy } \\
\hline Yes & 191 & 65.41 \\
\hline No & 101 & 34.59 \\
\hline \multicolumn{3}{|l|}{ Physiotherapy } \\
\hline Yes & 131 & 44.86 \\
\hline No & 161 & 55.14 \\
\hline
\end{tabular}

$(52.40 \%)$. The presence of pain in different joints, defined as polyarthralgia, was the most common ailment, along with the spine $(35.27 \%$ and $27.40 \%$, respectively). The presence of degenerative pathogenesis was statistically higher compared to traumatic pathogenesis $(67.47 \%)$, and $45.55 \%$ of the sample had already undergone surgery. Most of the subjects were already following a therapy prior to the lockdown $(65.75 \%)$.

\section{Physical Activity}

In terms of physical activity performed before and during the lockdown, what emerged was that this was 
significantly reduced when considering the totality of the sample ( $p<0.0001)$ [Table 2]. Stratifying the sample by age, this reduction was not significant only in subjects aged $<35$. Similarly, by stratifying the sample by chosen profession, the difference between the execution of physical activity before and during the lockdown in all the professions, except for students, was statistically significant $(p=0.65)$. The analysis of the data based on the anatomical area affected by the orthopaedic pathology showed a significant reduction in physical activity in all the categories, with the exception of upper limb and foot. In addition, the reduction in physical activity was statistically significant, regardless of sex, degenerative or posttraumatic etiopathogenesis, or whether the subject had undergone surgery or not.

\section{Couch Time}

Similarly, the increase in sedentary lifestyle was statistically significant throughout the whole sample $(\mathrm{p}<0.0001)$ with as many as 169 subjects $(57.88 \%)$ who spent more than 3 hours a day performing sedentary activities during the lockdown [Table 2]. By stratifying the sample for the aforementioned categories, the increase in couch time was not significant in the groups of subjects who are aged $<35$, students and subjects suffering from upper limb and foot pathologies. A sedentary lifestyle in patients with knee pathologies increased, but not in a statistically significant manner.

\section{Physiotherapy}

A total of 161 subjects (55.14\%) underwent regular or periodic physical therapy prior to the lockdown. During the lockdown, $87.58 \%$ of these had to suspend their rehabilitation cycles. However, 64 of the 141 people reported continuing self-managed physiotherapy at home. When comparing the pain level reported during the lockdown between those who continued self-managed physiotherapy and those who stopped it completely, no significant differences emerged $(5.1 \pm 2.4$ and $5.8 \pm 2.5$, respectively, $\mathrm{p}=$ 0.1286). The worsening of pain symptoms from before to during the pandemic was significant for both groups (0.0134 and 0.0079 , respectively).

\section{Therapy Adjustment}

A total of 191 people were taking or performing some pharmacological or physical therapy prior to the lockdown; during the lockdown, 231 subjects did not need to modify or begin therapy, 44 had to adjust their therapy and 17 had to suspend their intra-articular infiltrations.

The variation in therapy from before to during the lockdown is statistically significant throughout the entire sample ( $<<0.0001$ ) [Table 3]. However, by analysing the different stratifications of the sample individually, the variation in therapy is significant for both genders, though only significant in subjects between 35 and 70 years of age. Retired people and sedentary workers are the only occupational categories that needed a significant change in therapy ( 0.029 and $<0.0001$, respectively). With regard to pain location, only people with knee pathologies showed a significant difference $(p=0.0487)$. A significant variation of therapy was found in subjects affected by a degenerative pathology ( $p<0.0001)$ but not in those with post-traumatic pathologies. Significant variations regardless of prior surgeries.

\section{Pain}

The average NRS before the lockdown was $4.3 \pm 2.3$, which then became $5.2 \pm 2.5$ during the lockdown ( $p$ $<0.0001)$ [Table 3]. The perceived pain was statistically significantly increased regardless of gender, etiopathogenesis and prior surgeries. With regard to age, pain worsening was significant only in subjects between 35 and 70 years of age ( $p<0.0001$ ); with regard to professions, there was a significant deterioration only among manual and sedentary workers (0.0015 and 0.0009 , respectively). Only the subjects with polyarthralgia experienced a significant worsening of their pain $(p=0.0001)$. For patients who did not exercise during the lockdown, the average NRS value was $5.7 \pm 2.6$, while the average value in those who exercised was $4.5 \pm 2.4(\mathrm{p}=0.0001)$.

\section{Patients Self-Evaluation}

Figures 1 and 2 illustrate the distribution of responses regarding the last two, purely qualitative, questions of the questionnaire, which concern the opinion of the subjects who participated in the survey. A total of 157 people $(53.77 \%)$ believe they need an orthopaedic re-evaluation of their problem following the lockdown. Comparing the average pre-lockdown pain of those who deem a new orthopaedic visit necessary with those who do not, there were no statistically significant differences $(p=0.7072)$. On the other hand, when comparing the pain reported during the lockdown, there is a significant increase in NRS in those who believe they should undergo a new orthopaedic visit, compared to those who do not ( $p$ $<0.0001$ ) [Table 4]. 


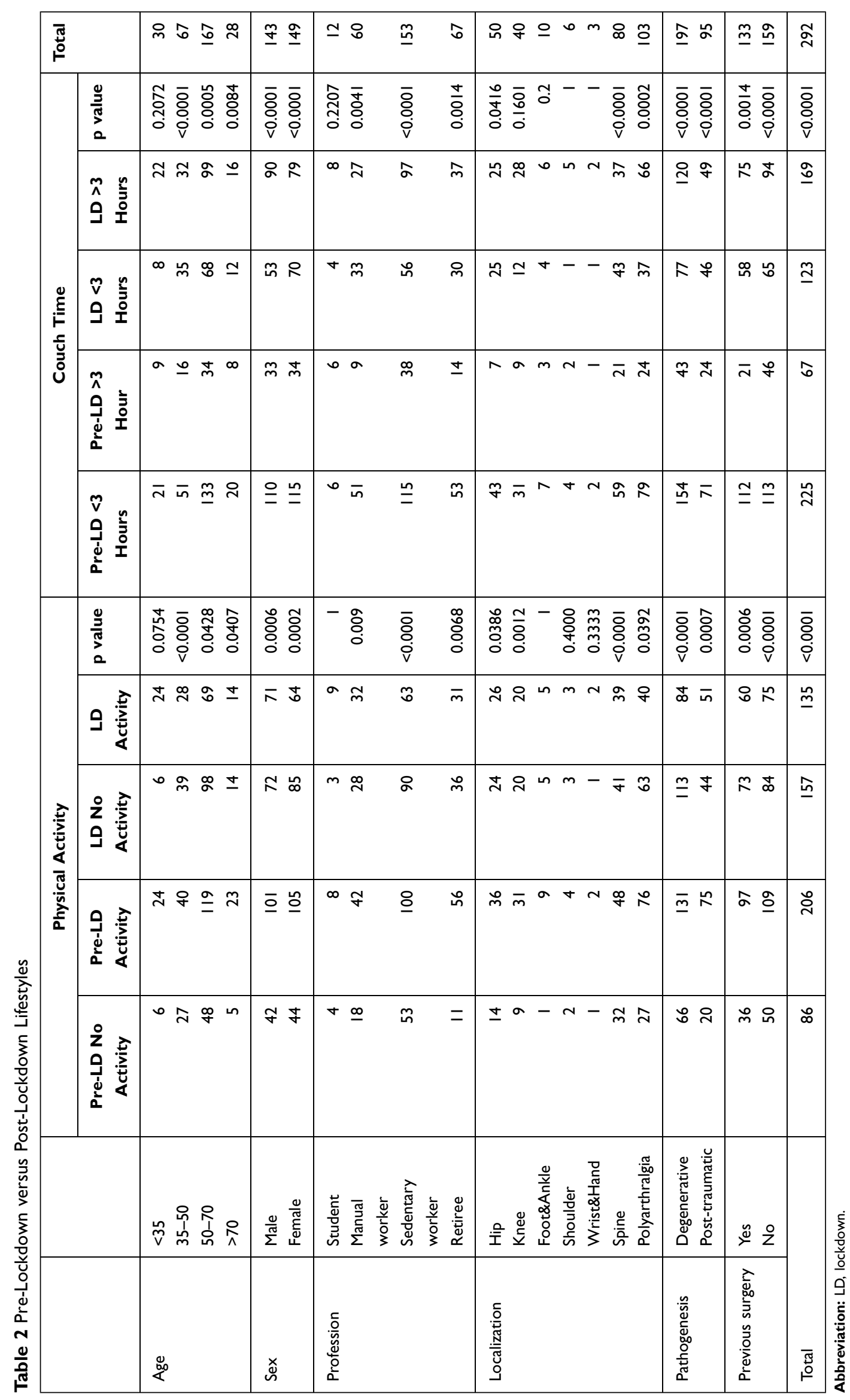




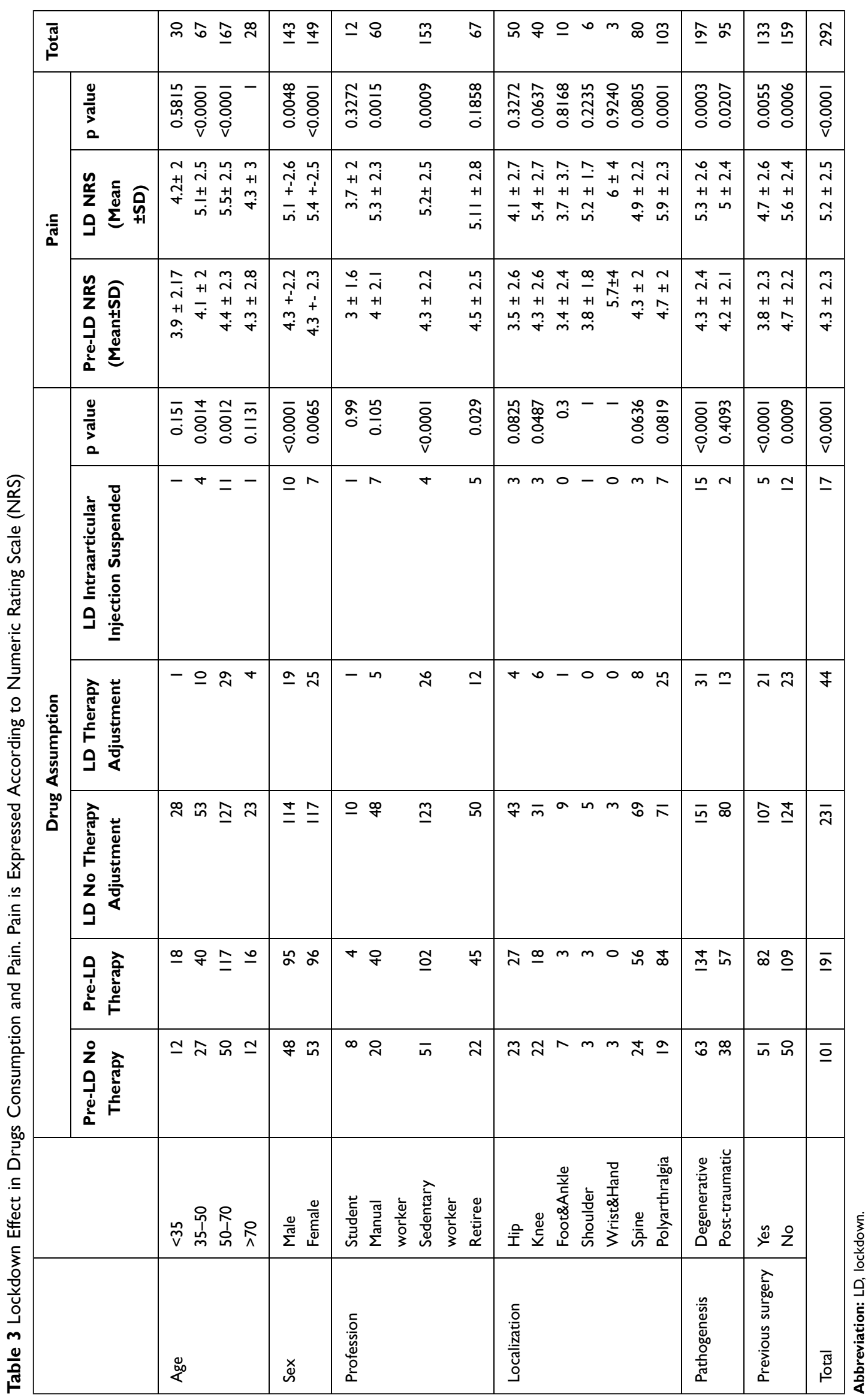


What impact did the lockdown had on your symptoms?

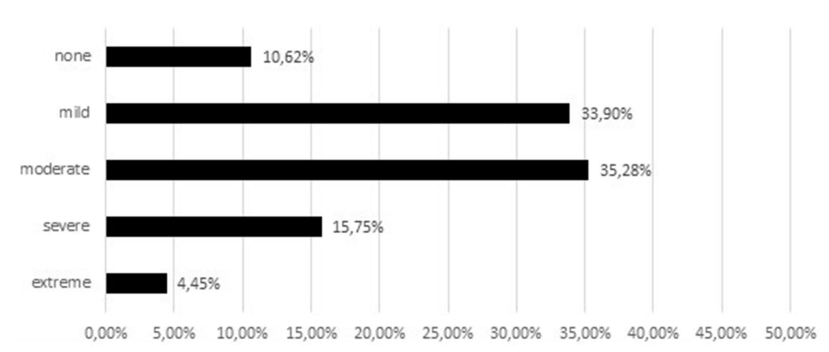

Figure I Qualitative question and answers of the survey about the perceived worsening of the symptoms due to lockdown.

When the lockdown will be over, will you go for an orthopaedic consultation?

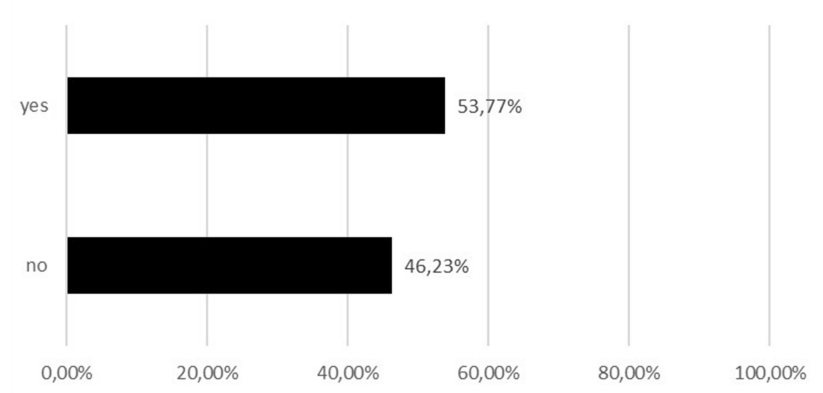

Figure 2 Qualitative question and answers of the survey about the perceived necessity of an orthopedic consultation after the end of the lockdown.

\section{Discussion}

Considering the totality of the sample, there was a reduction in the performance of sports activities during the lockdown, an increase in sedentary lifestyle, an increase in pharmaceutical drug intake and a reduction in physiotherapy performed, with an increase in perceived pain.

The change in lifestyle habits, in terms of reduction of physical activity and increase in couch time, was felt across the whole sample with the exception of patients aged $<35$ and students. One possible explanation could be the easier adaptability of younger subjects who, by

Table 4 Mean NRS Values Between Subjects Who Needs Orthopaedic Control Visit Pre-Lockdown and During Lockdown

\begin{tabular}{|l|l|l|l|}
\hline & Need for Check-Up & No Need & p value \\
\hline Pre-LD pain & $4.24 \pm 2.4$ & $4.3 \pm 2.2$ & 0.7072 \\
\hline Post-LD pain & $5.8 \pm 2.4$ & $4.4 \pm 2.4$ & 0.0001 \\
\hline
\end{tabular}

Abbreviation: LD, lockdown. using multimedia tools and supports, were able to more easily compensate for the imposed restrictions: for example, the massive use of technology to access online workout courses.

The orthopaedic patients who underwent variations in their therapy were 35-70 years of age. This centralised distribution can be explained by several factors. First of all, the adaptability of the younger age groups, as mentioned above. In the more advanced age groups, on the other hand, the presence of priorly set therapies, associated with the increased difficulty in accessing medical consultations and treatments, which during the lockdown were performed mainly online and therefore more difficult for people who were less accustomed to using these systems, may have constituted a second limit. The third possible explanation could lie in the fact that, prior to the lockdown, the working categories had a much more dynamic lifestyle than the non-working categories and therefore in proportion these subjects showed more evident changes, as also testified by the significant worsening of perceived pain in these categories when comparing young and old, students and retired people.

A worsening of pain symptoms emerged regardless of the pathogenesis and any prior surgeries.

The pain was significantly lower in those who exercised during the lockdown. According to the literature, complete inactivity along with an increase in time spent performing sedentary activities worsens the pain. ${ }^{12,16,17,19}$

No significant differences emerged between those who continued self-managed physiotherapy and those who stopped it completely, probably because performing physiotherapy without being followed by a specialist and without suitable equipment affects the result of the therapy itself.

However, with regard to the anatomical districts involved, only patients suffering from polyarthralgia experienced a significant worsening of pain. Among the subjects who had multiple affected anatomical districts, the areas of interest were mainly hip, spine and knee (221 subjects), in accordance with the results obtained in the literature, regarding the increase in pain in highly sedentary subjects. ${ }^{11}$

The mood-lowering during lockdown may also have played a role in the perception of pain in patients functionally more limited like polyarthralgic ones. Nevertheless, there was no section in our survey dedicated to evaluate the psychological area of the interviewees.

Tran et al believe that the restrictions made necessary to contain the COVID-19 pandemic have had a much 
deeper impact, one that goes beyond just lifestyle changes. The emotional, affective and psychological component are of primary importance, ${ }^{21}$ together with the economic one. ${ }^{5}$ Daily habits have therefore been deeply affected by this state of uncertainty and anxiety that the pandemic has brought about. ${ }^{20}$

An interesting Chinese study showed that during the outbreak of the pandemic, negative feelings of anxiety, depression and indignation gradually but visibly overtook positive emotions. At the same time, interest in topic such as health and family increased while interest regarding free time and friends decreased. ${ }^{14}$

A recent Italian study ${ }^{18}$ performed a review of the most recent literature in the field of psychological problems caused by a state of lockdown and isolation. Pervasive anxiety, uncontrolled fear of infections, but also frustration and boredom, are the most common feelings described by the population during the lockdown.

The limitations of this study are various. The representativeness of the sample was affected by the small sample size and by the methodology, on a voluntary and online basis, through which the questionnaire was administered.

In order to guarantee anonymity, sensitive data could not be collected; hence, the impossibility to recall the patients represents one of the limits of the study.

The elderly population was more difficult to reach probably due to a reduced familiarity with technology and difficulty in reading from electronic devices. Finally, having collected little data concerning people affected by pathologies of the upper limb (shoulder, wrist, hand) and foot, this may have undermined the statistical significance of the tests performed on these particular subgroups.

The strength of this study was the abundance of diversified data collected through which we were able to analyse various aspects related to both orthopaedic pathologies and the lockdown. To date, there are no other studies in the literature that have analysed the perception of orthopaedic pathologies during this exceptional period, which served as a laboratory of sorts in which the entire population was subjected to the same living conditions. The imposed lockdown was a necessary, exceptional and unprecedented measure, which highlighted how physical activity, freedom of movement and sociability impact the subjective perception of orthopaedic pathologies. Further studies would be necessary in order to quantify the importance of the psychological aspect of the lockdown on the perception of pain. The increased difficulty in accessing treatment for non-COVID diseases that emerged during the lockdown months may also have contributed to this.

\section{Conclusion}

During the lockdown, patients suffering from orthopaedic and traumatological pathologies experienced a worsening in their pain symptoms, a variation in the consumption of pharmaceutical drugs, a decrease in physical activity and an increase in sedentary lifestyle. The lockdown, a necessary measure of a completely exceptional character, has allowed us to deduce how much the social impact of the event and the sudden changes in lifestyle, inherent to physical/workrelated activity and one's psychological state, have negative effects on the perception of the orthopaedic pathology.

\section{Data Sharing Statement}

The data that support the findings of this study are available on request from the corresponding author.

\section{Ethical Review Committee Statement}

In light of the Italian law, authors are not required to ask for approval of an institutional review board or ethical committee for this type of study. However, data management and consent acquisition have been conducted in accordance with the European Union General Data Protection Regulation - GDPR 2016/679 - and in conformity with the principles of the Helsinki Declaration. This research was approved by our University and has been published and advertised on the institutional website: https://www.uniroma1.it/it/notizia/un-questionario-suiproblemi-ortopedici-nella-fase-del-lockdown

\section{Funding}

The authors received no specific funding for this work.

\section{Disclosure}

The authors declare that they have no competing interests.

\section{References}

1. Ali I, Alharbi OML. COVID-19: disease, management, treatment, and social impact. Sci Total Environ. 2020;725:138861. doi:10.1016/j. scitotenv.2020.138861

2. Amerio A, Bianchi D, Santi F, et al. Covid-19 pandemic impact on mental health: a web-based cross-sectional survey on a sample of Italian general practitioners. Acta Bio Medica. 2020;91(1):83-88. doi:10.23750/abm.v91i2.9619 
3. Angiocola PDL, Monti M. COVID-19: the critical balance between appropriate governmental restrictions and expected economic, psychological and social consequences in Italy. Are we going in the right direction?. Acta Bio Medica Atenei Parmensis. 2020;91:35-38.

4. Blustein DL, Duffy R, Ferreira JA, Cohen-Scali V, Cinamon RG, Allan BA. Unemployment in the time of COVID-19: a research agenda. J Vocat Behav. 2020;119:103436. doi:10.1016/j.jvb.2020. 103436

5. Brenner MH. Will there be an epidemic of corollary illnesses linked to a COVID-19 - related recession? Am J Public Heal. 2020;110 (7):974-975. doi:10.2105/AJPH.2020.305724

6. Collivignarelli MC, Abbà A, Bertanza G, Pedrazzani R, Ricciardi P, Carnevale Miino M. Lockdown for CoViD-2019 in Milan: what are the effects on air quality? Sci Total Environ. 2020;732:139280. doi:10.1016/j.scitotenv.2020.139280

7. Crawley E, Loades M, Feder G, Logan S, Redwood S, Macleod J. Wider collateral damage to children in the UK because of the social distancing measures designed to reduce the impact of COVID-19 in adults. BMJ Paediatr Open. 2020;4(1):e000701.

8. Goethals L, Barth N, Guyot J, Hupin D. Impact of home quarantine on physical activity among older adults living at home during the COVID-19 pandemic: qualitative interview study corresponding author. JMIR Aging. 2020;3:1-5.

9. Gorbalenya AE, Baker SC, Baric RS, et al. The species Severe acute respiratory syndrome-related coronavirus: classifying 2019-nCoV and naming it SARS-CoV-2. Nat Microbiol. 2020;5(4):536-544.

10. Grasselli G. Clinical care utilization for the covid 19 outbreak in lombardy. N Engl J Med. 2020;19:1-2.

11. Kim SD. Association between sitting time and orthopedic conditions in Korean older adults. Geriatr Nurs. 2019;40(6):629-633. doi:10.1016/j.gerinurse.2019.06.007

12. Lee J, Chang RW, Ehrlich-jones L, Kwoh CK, Nevitt M, Semanik PA. Sedentary behavior and physical function: objective evidence from the osteoarthritis initiative. Arthritis Care Res. 2016;67(3):366-373. doi:10.1002/acr.22432
13. Li L, Li Q, Huang L, et al. Air quality changes during the COVID-19 lockdown over the Yangtze River Delta Region: an insight into the impact of human activity pattern changes on air pollution variation. Sci Total Environ. 2020;732:139282. doi:10.1016/j.scitotenv.2020.139282

14. Li S, Wang Y, Xue J, Zhao N, Zhu T. The impact of COVID-19 epidemic declaration on psychological consequences: a study on active Weibo users. Int J Environ Res Public Health. 2020;17 (6):2032. doi:10.3390/ijerph17062032

15. Narici M, De Vito G, Franchi M, et al. Impact of sedentarism due to the COVID-19 home confinement on neuromuscular, cardiovascular and metabolic health: physiological and pathophysiological implications and recommendations for physical and nutritional countermeasures. Eur J Sport Sci. 2020:1-22. doi:10.1080/17461391.2020.1761076

16. Park SM, Kim HJ, Jeong H, et al. Longer sitting time and low physical activity are closely associated with chronic low back pain in population over 50 years of age: a cross-sectional study using the sixth Korea National Health and Nutrition Examination Survey. Spine J. 2018;18(11):2051-2058. doi:10.1016/j.spinee.2018.04.003

17. Senba E, Kami K. Neurobiology of Pain A new aspect of chronic pain as a lifestyle-related disease. Neurobiol Pain. 2017;1:6-15. doi:10.1016/j.ynpai.2017.04.003

18. Serafini G, Parmigiani B, Amerio A, Aguglia A, Sher L, Amore M. OUP accepted manuscript. Qjm an Int J Med. 2020;1-7.

19. Stefansdottir R, Gudmundsdottir SL. Sedentary behavior and musculoskeletal pain: a five-year longitudinal Icelandic study. Public Health. 2017;149:71-73. doi:10.1016/j.puhe.2017.04.019

20. Torales J, Higgins MO, Castaldelli-Maia JM, Ventriglio A. The outbreak of COVID-19 coronavirus and its impact on global mental health. Int J Soc Psychiatry. 2020;3-6.

21. Tran LD, Rice TH, Ong PM, Banerjee S, Liou J, Ponce NA. Impact of gentrification on adult mental health. Health Serv Res. 20 20;36:90-94.

22. Wang C, Horby PW, Hayden FG, Gao GF. A novel coronavirus outbreak of global health concern. Lancet. 2020;395(10223): 470-473. doi:10.1016/S0140-6736(20)30185-9
Journal of Pain Research

\section{Publish your work in this journal}

The Journal of Pain Research is an international, peer reviewed, open access, online journal that welcomes laboratory and clinical findings in the fields of pain research and the prevention and management of pain. Original research, reviews, symposium reports, hypothesis formation and commentaries are all considered for publication. The manuscript management system is completely online and includes a very quick and fair peer-review system, which is all easy to use. Visit http:// www.dovepress.com/testimonials.php to read real quotes from published authors. 